XXI.

\title{
Die Verwendung der Klemmtechnik in der Chirurgie, nach dem Prinzip der fremdkörperfreien Klemmnaht. \\ Von
}

\author{
Dr. Ferd. Schultze in Duisburg. \\ Chirurg. Oberarzt am St. Vincenz-Hospital. \\ Vortrag gehalten auf der Versammlung deutscher Naturforscher und Ärzte \\ in Meran 1905.
}

(Mit 16 Abbildungen.)

Auf der Versammlung deutscher Naturforscher und Ärzte zu Aachen im Jahre 1900 berichtete ich an dieser Stelle über eine neue Nahtmethode, die Klemmnaht.

Die Technik derselben besteht in der Fixation der Wundränder mittels Roserscher Klauenschieber in Abständen von zirka 1/2 $\mathrm{cm}$, in der Anlage eines gewöhnlichen Verbandes und Entfernung der Schieber direkt nach Fertigstellung des Verbandes. Zweck dieser Methode ist Ausschaltung der eventuellen Stichkanaleiterung.

Indiziert ist die Klemmnaht überall da, wo man die gewöhnliche Hautnaht zu machen pflegt, Spannungen der Haut sind selbstredend ausgeschlossen, ebenfalls bei Parallelschnitten kann dieselbe keine sichere Verwendung finden. Die exakte Blutstillung ist eine Notwendigkeit, eventuell ist eine Drainage von der Wunde aus oder durch Knopfloch vorzunehmen. An den Extremitäten wird zur Sicherung stets ein Schienenverband angelegt.

Auf die einzelnen Applikationen der Klemmnaht will ich nicht näher eingehen, weil alles dem vorliegenden Schema zu entnehmen ist, nur möchte ich kurz betreff der Bauchnaht erwähnen, daß ich dieselbe bei seitlicher oder medianer Schnittführung in den Indikationenkreis gezogen habe. Es ist nach vorher ausgeführter exakter Etagennaht die Klemmnaht stets mit bestem Frfolg ans- 
zuführen. Nur bei Operationen der Rückenzone habe ich die Methode nicht verwendet.

Um die Nahtmethode mit Erfolg ausführen zu können, sind notwendig:

1. Roser sche Klauenschieber, nie Péau, weil derselbe nicht federt,

2. normale Elastizität der Haut,

3. Blutstillung,

4. exakter Verband mit Binde oder Kollodium (Fig. 1, 2 п. 2a).

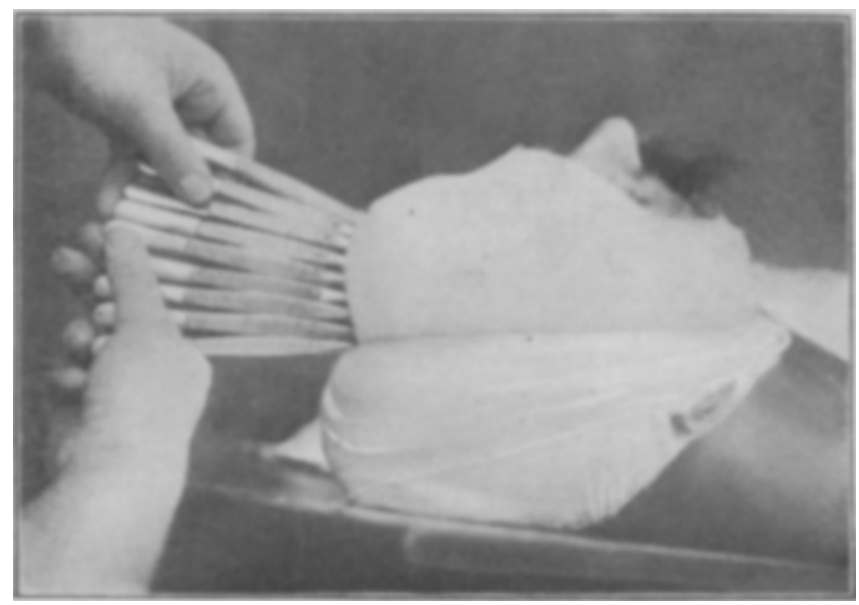

Fig. 1.

Die vorstehend geschilderte Klemmtechnik habe ich nun in der operativen Chirurgie weitgehend ausgenutzt. Der Klemmnaht fällt aber hier die Aufgabe zu, als vorbereitende Naht zu dienen.

Meine ersten Mitteilungen über diese Methode machte ich 1904 auf dem Kongreß für orthopädische Chirurgie, weiterhin auf der Versammlung deutscher Naturforscher und Ärzte in Breslan 1904. Im Juli 1905 berichtete Bernhard-Samaden ebenfalls über dieselbe voll mir empfohlene Methode der Klemmtechnik. Die Idee sowohl als auch die Technik deckt sich absolut mit den früher von mir gemachten Mitteilungen. Die Art des Instrumentes spielt hier keine Rolle. Sehr gut sind klanenförmige Péaus in verschiedener Stärke.

Die Technik ist eine äußerst einfache. Es werden mittels Klauenschieber die Wundflächen, welche vereinigt werden sollen, fixiert. Nach dieser Fixation ist das ganze Gebiet nahtgerecht, zwischen jeden Klanensehieber wird eine Naht gelegt. Handelt es 
Die Verwendung der Klemmtechnik in der Chirurgie usw.

sich um Nahtanlagen in Körperhöhlen, so ist eine Extension an den Klauenschiebern notwendig, um jedesmal neben demselben eine

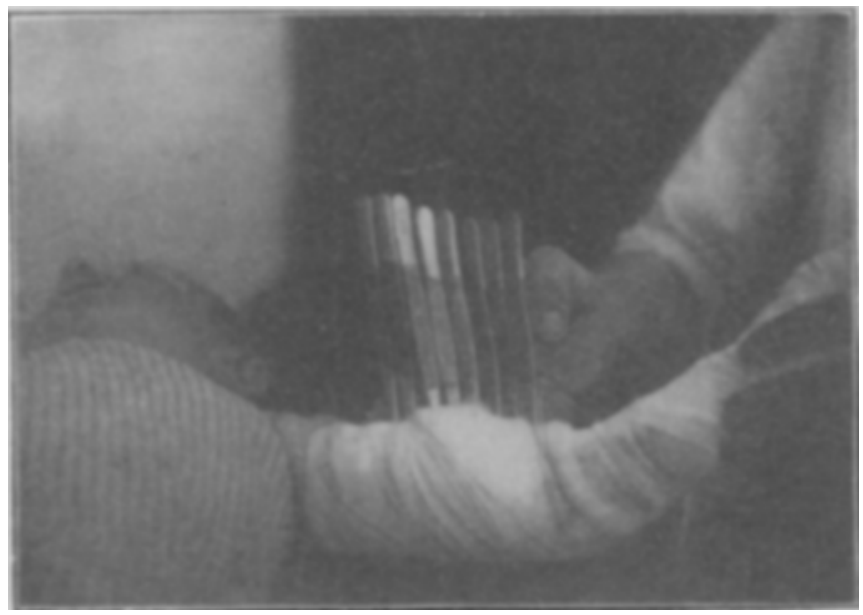

Fig. 2.

Naht anzulegen. Gerade bei Nähten in Körperhöhlen ist der Nutzungswert dieser Naht nicht zu unterschätzen.

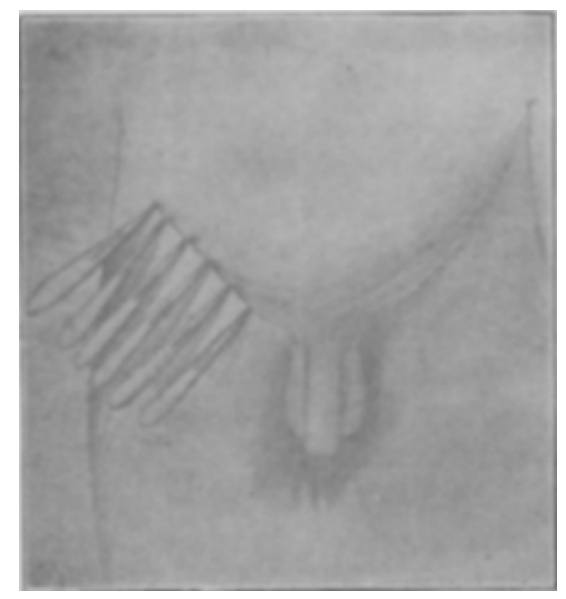

Fig. 2a.

Hat man sich mit der Technik vertrant gemacht, so findet man Gelegenheit, täglich davon Gebrauch zu machen, sei es um einen 
temporären Abschluß der Wunden zu erreichen, sei es um an irgendwelchen Stellen des Körpers einen Verschluß durch die Seidenoder Katgutnaht zu erzielen.

Was nun den temporären Verschluß angeht, so kann derselbe zur Blutstillung verwendet werden. Zu diesem Zweck wird die Wunde austamponiert und dann mittels Klauenschieber geschlossen.

Von wesentlicher Bedeutung ist die Übertragung der Klemmtechnik auf die gewöhnliche Naht. Überall da, wo wir zu nähen pflegen, kommt diese Technik in Frage. Die Vorzüge bestehen in der Zeitersparnis sowie in der Vereinfachung der Naht, ferner in der Erhöhung der Aseptik, da große Wundflächen rasch abgeschlossen werden können. Die absolut sichere exakte Adaption der Wundränder sowie die Möglichkeit, das feinste Nahtmaterial zu verwenden, dürfte als ein besonderer Vorteil bezeichnet werden.

Um auf die weitgehende Verwendung dieser Methode einzugehen, muß ich kurz auf die einzelnen Operationen hinweisen. Wir wollen dieselben einteilen in Operationen an den äußeren Bedeckungen und Operationen an den inneren Organen.

Die Operationen der äußeren Bedeckung sollen sich beziehen auf Haut, Muskel, Sehne, Nerv und Gefäße. Die Haut wird geklemmt and mit feiner Seide genäht; selbst da wo größte Spannung ist, kann man mit diesem feinen Material arbeiten. Eine größere Erleichterung gibt die Klemmtechnik bei den plastischen Operationen und Transplantationen sowohl im Gesicht als auch in den übrigen Körperzonen. Bei der Plastik wird der Lappen verlagert, geklemmt, genäht, bei der Transplantation erfolgt die Naht des aufgelegten Lappens, nachdem derselbe vorher unter bestimmter Spannung festgeklemmt wurde. Die Hautwunden geben wohl am häufigsten Gelegenheit zur Verwendung der Klemmtechnik. Es erfordert stets nur einen kurzen Moment, um den Schluß der Wunde zu erreichen. Als Nahtmaterial wird feinste Seide gebraucht, Nr. 0-1, die Fäden werden lang abgeschnitten, damit man dieselben später leichter entfernen kann. Die fortlaufende Naht mit dem feinen Faden ist unzweckmäßig, weil die Entfernung der Naht wegen des dünnen Materials schwierig ist. Um dies jedoch zu ermöglichen, kann man einen Längsfaden, welcher eingenäht wird, auflegen. Bei Entfernung der Naht erreicht man dann durch Anspannung dieses Längsfadens leicht seinen Zweck.

Bei der Muskel-Sehnenplastik (Fig. 3, 4) möchte ich die Klemmtechnik nicht mehr entbehren, sei es, daß es sich um Verkürzung oder Verlängerung der Muskeln und Sehnen, sei es, daß es sich um 
die Übertragung von einem Muskel auf den anderen handelt. Selbstverständlich überträgt sich diese Technik auch auf die Verletzungen der Sehnen, sowie auf die damit event. verbundenen Plastiken der Muskeln. Der klauenförmige Péau leistet hier vorzügliche Dienste.

Die Nervenplastik sowie die Nervennaht gestatten eine vorteilhafte Verwendung der Klemmtechnik.

Dasselbe dürfte gelten von einer eventuellen Naht der Gefäße. Ich bin zwar noch nicht in der Lage gewesen, dies auszu-

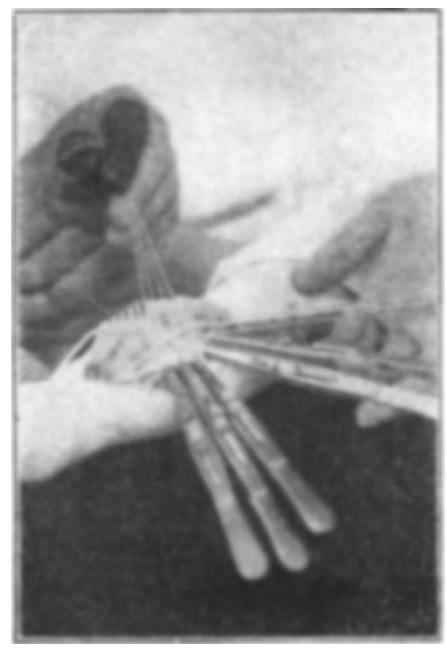

Fig. 3.

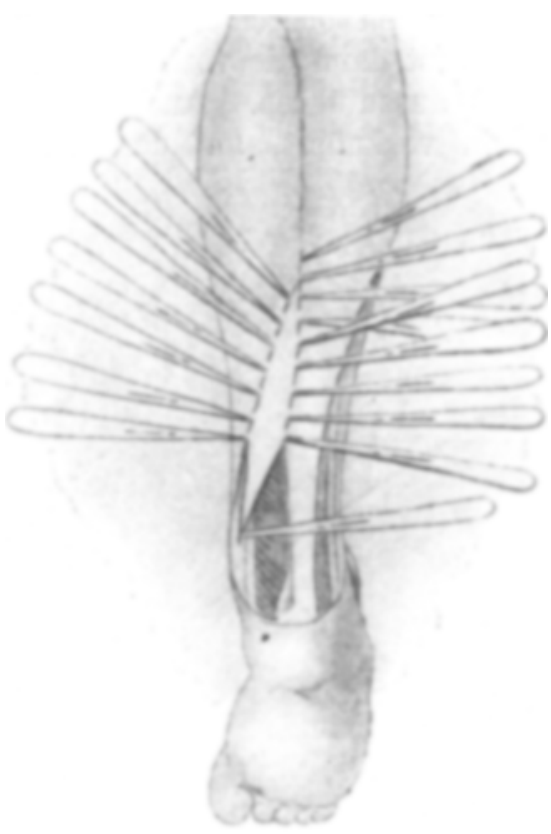

Fig. 4.

nutzen. Feineren Klauenschiebern dürfte anch hier der Vorzug zu geben sein.

Am Kopf finden wir ausgiebige Verwendung unserer Methode, bei den Plastiken, der Hasenscharte, der Gaumenspalte, bei den Resektionen der Kiefer- und Zungenoperationen. Auf die Gesichtsplastik brauche ich nicht weiter einzugehen, da sich alles nach den allgemein gültigen Grundsätzen hier regelt, dasselbe gilt von der Hasenscharte. Bei der Operation der Gaumenspalte bedeutet es eine wesentliche Vereinfachung der Technik sowie eine bessere Prognose, wenn man methodisch die Wundränder mittels der 
Klanenschieber fixiert und so durch eine exakte Adaption das ganze Terrain nahtgerecht macht (Fig. 5). Die Operation wird folgendermaßen ausgeführt. Nach Erledigung der üblichen Voroperation, welche die genügende Mobilisation des Gaumens garantiert, wird die Uvula sowie der weiche Gammen durch 2--3 Klanenschieber fixiert. Man erleichtert sich die Technik dadurch, daß man die Gaumendecken an der Übergangsstelle zmm weichen Gaumen grob mit einem Péau faßt und so ungefähr senkrecht in den Mund hineinstellt. Zweckmäßig muß der Péau oder auch Klatenschieber am oberen Ende rechtwinklig gebogen sein. Auf diest Weise ist dann die vorbereitende Klemmnaht des weichen Gaumens leicht auszuführen. Auch ist es nicht mit besonderen Schwierigkeiten verknüpft, auf der hinteren Seite des weichen Gaumens
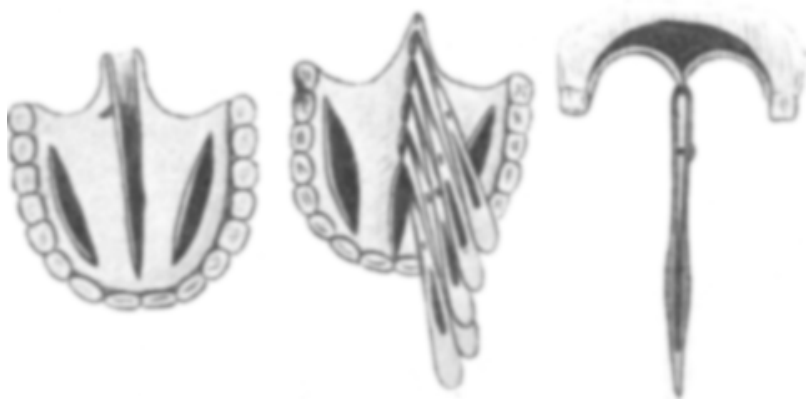

Fig. 5.

Nähte anzubringen, wodurch die Sicherheit des Resultates noch erhöht wird. Die Naht, besonders an der Übergangsstelle vom weichen zum harten Gaumen, muß unter exakter Adaption, möglichst mit steilgestellten Wundrändern gemacht werden. Ein Vorzug der ganzen Methodik liegt bei der ganzen Gaumenspaltoperation schon darin, daß man die Naht bequem mit dem gewöhnlichen Nadelhalter anlegen kann. Man arbeitet gewissermaßen zutage, indem man mit dem Klaueuschieber sich das nahtgerechte Gaumenmaterial bequem an die Oberfläche zieht.

Analog pflegen sich die Verhältnisse nach temporärer Resektion, oder nach radikaler Resektion der Ober- und Unterkiefer zu gestalten. Wenn es hier gilt, am Gaumen oder am Zungengrund den notwendigen Abschluß durch die Schleimhautnaht zu machen, so werden wir in der Klemmnaht eine wesentliche Unterstützung finden. Dasselbe ist der Fall bei Operationen an der Zunge, welche nicht 
selten zu tief nach der Zungenbasis gelegenen Nähten Veranlassung: geben.

Noch ganz besonders will ich hier im Gesicht auf die Verwendung transplantierter Lappen hinweisen. Dieselben werden möglichst so groß geschnitten, daß dieselben z. B. eine exstirpierte Wange bedecken. Durch Klauenschieber wird der Lappen fixiert und tunlichst in seinem alten Elastizitätskoeffizienten - nicht zu stark - eingespannt und dann eingenäht. (Fig. 6). Abgesehen von der vereinfachten Technik hat diese Methode den Vorzug, daß glatte transplantierte Flächen resultieren.

Der Hals bietet für die Klemmtechnik kein besonderes Material, es sei denn, daß es sich darum handelt. den Ösophagus zu nähen. Bei

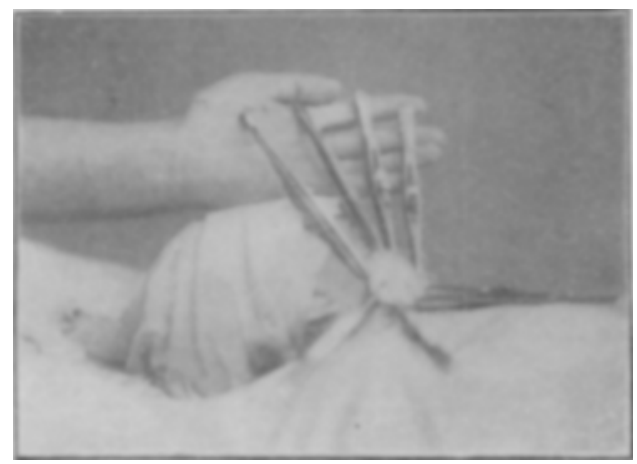

Fig. 6 .

der Kehlkopfexstirpation finden wir allerdings bei Ausführung der abschließenden Naht, wodurch die Speiseröhre von der Wundhöhle getrennt wird, eine nicht geringe Erleichterung.

Der Thorax gibt uns ebenfalls wenig Gelegenheit, unsere Technik zu verwenden, nur bei Verletzungen des Herzens wird uns dieselbe willkommen sein. Ich habe auch hier noch nicht das Glück gehabt, mich chirurgisch zu betätigen, zweifle jedoch nicht daran, daß man nach Anlegen der Klauenschieber die Blutung gestillt hat und alsdann die Naht leichter ausführen kann.

Wir kommen nun zur kurzen Besprechung der Operationen der Bauchhöhle. Hier finden wir ein reiches Feld für die Verwendung unserer Technik. In Betracht kommen die Operationen am Magen, am Darm, an der Gallenblase, ferner die Adnexoperationen, dann die Operationen der Bauchbrüche. Die Operationen am Magen 
finden eine ausführliche Darstellung in meiner Arbeit, Modifikation der Kocherschen Magenresektion (Dentsche Zeitschrift f. Chirurgie

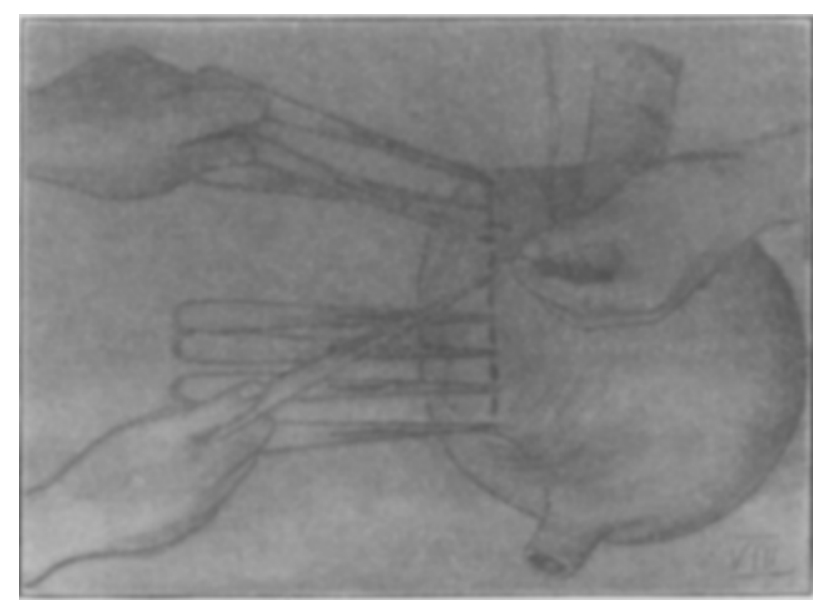

Fig. 7.

1905). Die Klemmtechnik ergibt sich hier von selbst und bedarf keiner weiteren Auseinandersetzung.

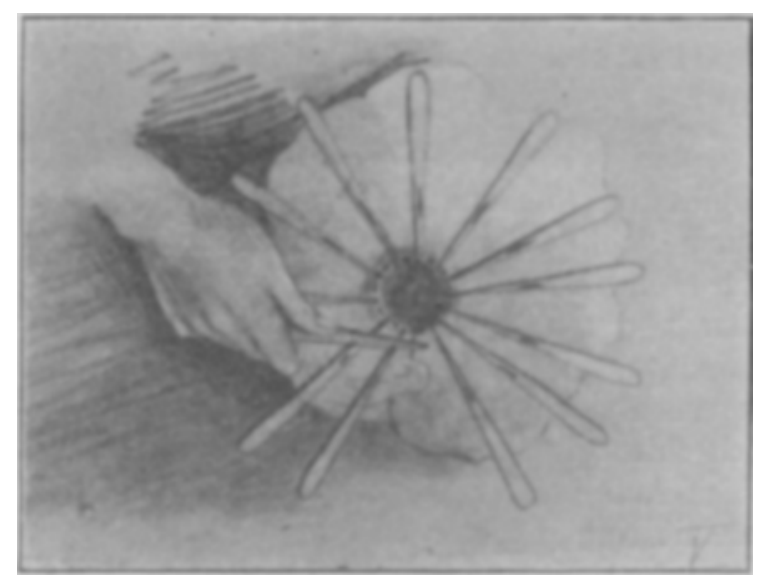

Fig. 8.

Der Verschlnß einer Magenwunde wird in der Weise gemacht, daß man die ganze Wunde nit Klauenschiebern zuklemmt und 
mit der fortlaufenden Naht versorgt. Alsdann wird die Serosa geklemmt und ebenfalls vernäht. Nach diesem Schema wird der Magen bei der Resektion des Pylorus versorgt, so bei der Segmentresektion, sowie bei der Pyloroplastik. (Fig. 7, 8.)

Etwas abweichend muß man bei der Gastroenterostomie, sowie bei der Darmnaht verfahren. Hier wird von innen geklemmt und von innen genäht. Die Gastroenterostomie ist in folgender Weise auszuführen: Klemmnaht der Serosa in gerader Linie mit an-

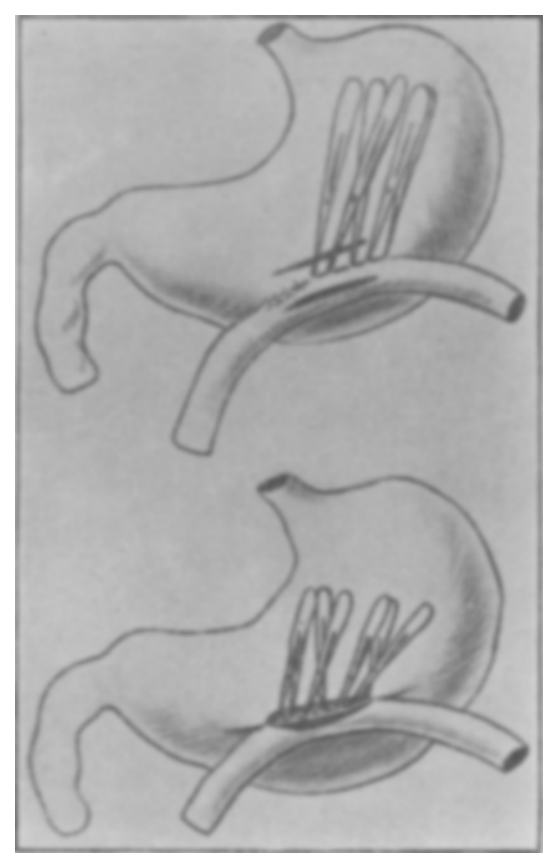

Fig. 9.

schließender fortlaufender Naht. So wird die Verbindung bis anf $1 / 3$ - $1 / 4$ geschlossen. Der Rest muß durch äußere Serosanähte einen festen Abschluß erreichen. Dadurch, daß man die Klauenschieber. nachdem bereits ein großer Teil der Peripherie genäht ist, hervorzieht, kann man sehr weit die innere Naht ausführen. (Fig. 9.) Die Serosanaht wird von außen noch hinzugefügt, soweit dieselbe noch nicht vorhanden war.

In analoger Weise gestaltet sich die Ausführung der Darmnaht. (Fig. 10). Nehmen wir z. B. eine größere Darmresektion 
an, so vollzieht sich dieselbe folgenderweise. Nach der üblichen Resektion wird das Mesenterium geklemmt und genäht, ebenso der Darm, so daß kaum eine kleine Öffnung übrig bleibt. Die Wandung, Schleimhaut und Serosa werden ganz durchstochen und die Serosanaht hinzugefügt, wodurch dann auch der letzte Verschluß des Darmes erledigt wurde. Auch bei den Totalexstirpationen des Magens möchte es eine wesentliche Erleichterung für die Operation sein, durch die exakte Klemmmethode sich die Naht vorzubereiten, um dann analog der Darmresektion die Technik zu erledigen.

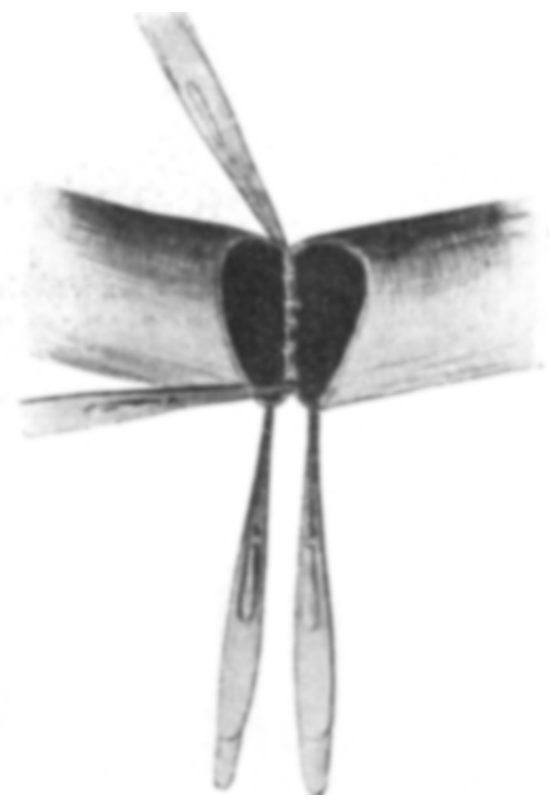

Fig. 10.

Die Operation des Anus praeternaturalis erfordert noch eine kuxze Anseinandersetzung, sei es, daß es sich um den definitiven oder den provisorischen Anus handelt. Bevor man die Eröffnung des Darmes vornimmt, ist ein exakter Bauchabschluß zu erstreben. Beim provisorischen Anus wird man nach Abschuüren Klemmen anlegen nnd den Peritonäalabschluß exakt herstellen. Hat man nun vorher z. B. die Darmklemme, welche am oberen Mundwinkel heraussieht, angelegt, so kann Darmöffnung, sowie Verbindung mit der äußeren Hant erfolgen, ohne daß der Darminhalt die Wunde überschwemmt. Beim definitiven Anus wird nach Fixation des Peritonäums der 
Darm isoliert, hervorgezogen peripher und zentral abgeklemmt. Beide werden zirkulär mit dem Peritonäum vernäht. Etagennaht der zwischen beiden Rohren gelegenen Brücke, Naht des Darmrohrs mit der Haut, Entfernung der Darmklemme vor Anlage der letzten Naht.

Um kurz hier auf die Operationsmethode des Wurmfortsatzes (Fig. 11) zurückzukommen, so ist die Klemmtechnik wohl bei einem Organ, welches so hervorragend im Vordergrund allgemeiner In-

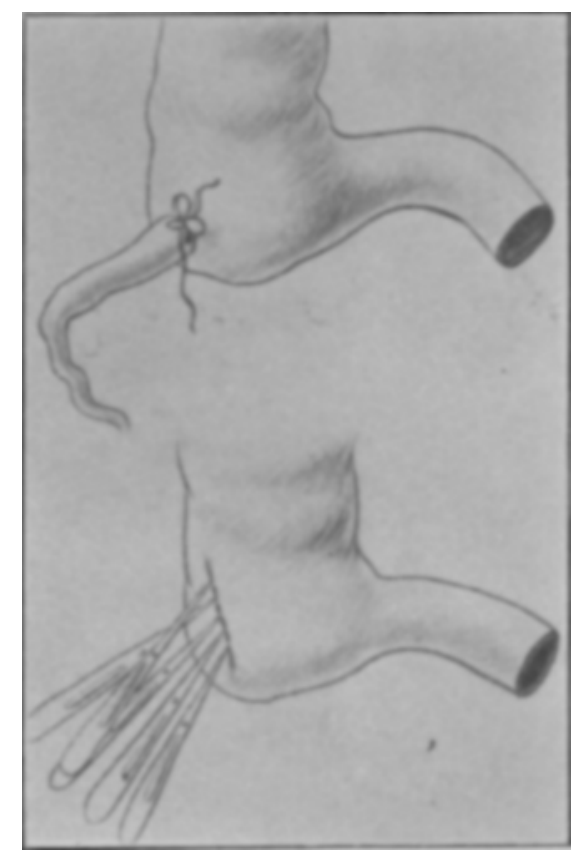

Fig. 11.

teressen steht, von nicht zu unterschätzender Bedeutung. Die Technik ist folgende: Abklemmung im Cöcum, Unterbindung des Wurmfortsatzes, des Mesenteriolum an der Radix, Klemmnaht der Serosa mit folgender Knopf- oder fortlaufender Naht. Der Abschluß ist ein sehr präziser und dauernd exakter.

Wir kommen nun zu den Operationen am Mastdarm. Hier handelt es sich um Entfernung größerer oder kleinerer Teile des Mastdarmes, Hämorrhoiden, Stenosen, Tumoren, welche unter Hilfe der Klemmtechnik manche Schwierigkeit aus dem Wege räumen. 
Die Operation der Hämorrhoiden beispielsweise (Fig. 12) wird folgenderweise ausgeführt: Ziskumzision am Anus unter Freilegung des M. sphincter, mit folgendem Abschluß des Darmrohres durch eine dicke Seidennaht, welche zugleich als Extensionszügel dient. Nach genügender Lösung trägt man Schnitt für Schnitt den vorgezogenen Darm ab, indem man zu gleicher Zeit Schleimhaut mit äußerer Haut durch Klauenschieber verbindet. Die Blutung wird dadurch gestillt; das exakt adaptierte Operationsfeld kann direkt durch die

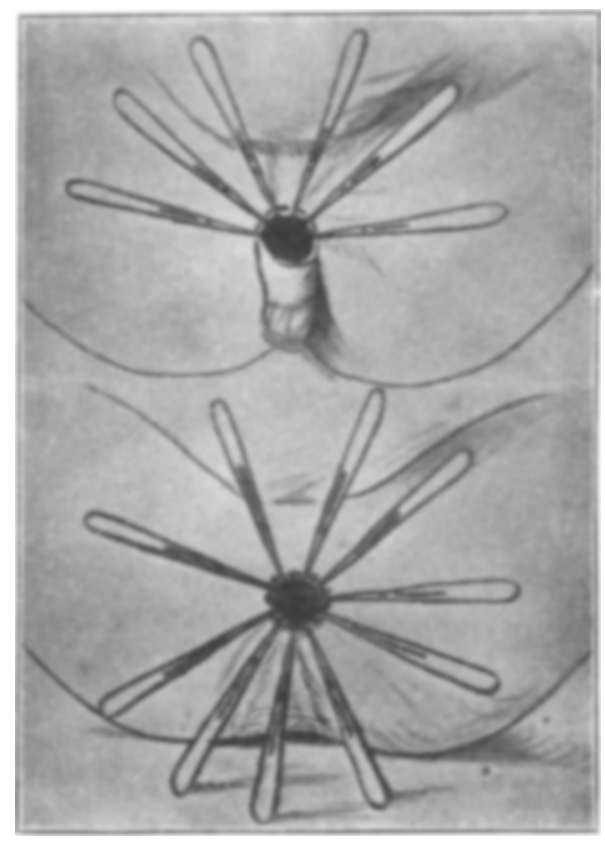

Fig. 12.

Naht fixiert werden. Bei Exstirpation des unteren Rektalendes wegen Tumoren wird man sich analog verhalten. Handelt es sich bei Erhaltung des unteren Rohres mit dem M. sphincter um die nun folgende Verbindung der beiden Darmenden, so reguliert sich hier die Naht wie oben erwähnt. Vom Darmlumen aus wird die ganze Peripherie geklemmt und genäht. Durch Extension am Klauenschieber kann man sich das Anlegen der Naht einfacher gestalten.

Die Gallenblase gehört anch zum Indikationskreis der Klemm- 
naht (Fig. 13). Bei sehr vergrößerter Gallenblase können wir bequem extraperitonäal arbeiten, wenn wir das Peritonäum der Gallenblase mittels Klemme mit der Haut verbinden. In kurzer Zeit ist dieser Peritonäalabschluß hergestellt, die Eröffinung der Gallenblase kann gemacht werden. Nachdem dann sofort die Wundränder der Blase mit denen der Haut geklemmt sind, ist die Gallenfistel fertig, um. bequem eine Abtastung mit dem Finger zu gestatten. Ist die Ausräumnng erfolgt, so können nach erfolgter regulärer Bauchtamponade nunmehr die Gänge in aller Ruhe abgesucht werden. Auch der Eröffnung des Ductus choledochus, sowie des cysticus leistet der Klauenschieber gute Dienste, ebenso bei der Cholecystektomie; letztere Operation bietet dem Klauenschieber günstige Gelegenheit, die Peritonäalnaht auszuführen.

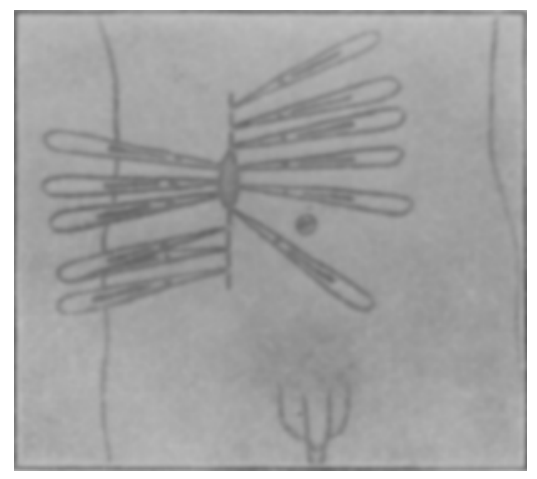

Fig. 13.

Was num die Adnexoperationen, Exstirpationen von Ovarialcysten usw. angeht, so bieten dieselben ebenfalls ein dankbares Material. Es gilt dies zugleich von den Cysten, welche sich im Mesenterium lokalisiert haben. Die Entfernung einer Ovarialcyste geht z. B. folgenderweise vor sich. Punktion, Abklemmungen der Wurzel mit breiter Zange, Abpräparieren eines Peritonäaltrichters, Entfernung der Cyste, Unterbindung der Gefäße, Naht der Peritonäaltrichters unter Hilfe der Klemmnaht. Auf diese Weise wird die dicke Umstechungsligatur mit Sicherheit vermieden und nur feinste Seide versenkt. Weiterhin verweise ich noch auf die plastischen Operationen der Vagina sowie auf die Dammplastik.

Nicht möchte ich nnerwähnt lassen, daß die Zerreißung des Peritonäum parietale am Beckenboden nach komplizierten Adnex- 
operationen unter dem Einfluß der Klemmtechnik in kurzer Frist wieder genäht werden kann, um so den unausbleiblichen Verwachsungen den Boden zu entziehen.

Bei den Operationen am uropoetischen Apparat wird die Technik durch Ausnutzung des Klauenschiebers wesentlich vereinfacht, so bei den Eingriffen an der Niere, am Ureter, an der Blase.

In der gestrigen Sitzung schlug v. Hacker die Mobilisation der Urethra vor nach Fxstirpation von Strikturen. Hier findet man ein geeignetes Feld, durch die Klemmnaht exakt die Rohrleitung

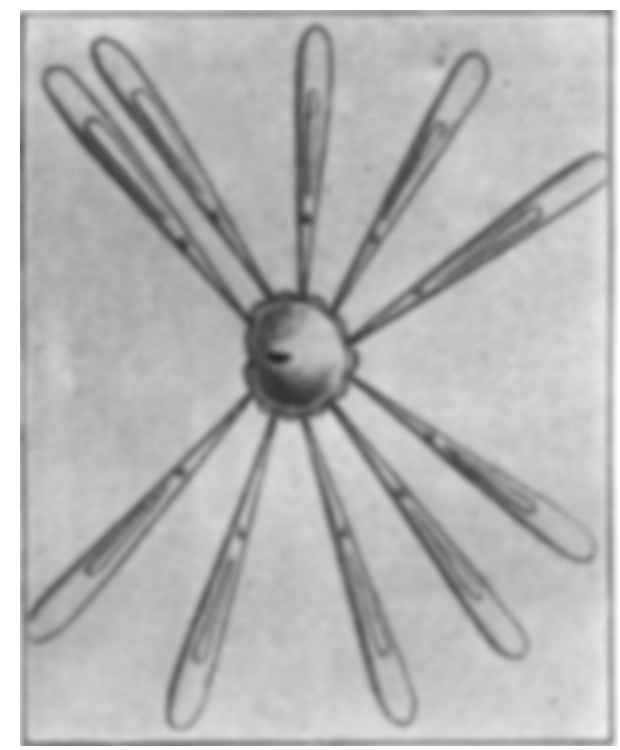

Fig. 14 .

wieder herzustellen. Die plastischen Operationen bei der Hypospadie will ich noch als besonders geeignet erwähnen, ebenso die Phimoseoperation (Fig. 14).

Zum Schluß kommen wir noch zur Besprechung der Hernien. Zur Vermeidung derselben pflegt man bei Laparotomien eine exakte Etagennaht anzulegen. Dieselbe gestaltet sich unter Zuhilfenahme des Klauenschiebers folgendermaßen. Zunächst wird das Peritonäum geklemmt und dann genäht; es folgen nach demselben Schema die einzelnen Schichten. Die Hautwunde wird entweder mit feinster Seide oder auch durch die typische fremdkörperfreie Klemmnaht geschlossen. 
Was die Laparotomie angeht, so mache ich seit einiger Zeit die Öffnung durch Öffnung der Rektusscheide. Letztere wird vorn gespalten, der Muskel beiseite gezogen, um die Spaltung der hinteren Scheide quer vornehmen zu können. Der Bauchschluß erfordert dann die Naht der hinteren Scheide, Reposition des Muskels sowie Naht der vorderen Scheide. Handelt es sich nun um Hernien, so sucht man nach Präparation des ganzen Feldes die Naht des Peritonäum zu erreichen. Dies gelingt umso leichter, wenn man unter Hilfe von Klauenschiebern die Peritonealhöhle abschließt und dann vernäht. Die übrigen nun folgenden Schichten werden ebenfalls geklemmt und genäht. Man wird stets dieselbe Beobachtung machen und erstaunt sein, wie trotz der großen Diastasen mit Leichtigkeit der Abschluß zustande kommt. Es gilt dies sowohl von den Hernien

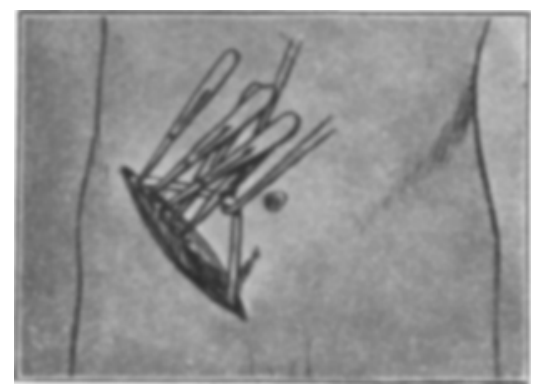

Fig. 15.

in der Mittellinie, als auch ganz besonders von denen in der Leiste. (Fig. 15.)

Diese in kurzen Zügen gehaltene Darstellung wird genügen, um sich von der Leistungsfähigkeit der Klemmnaht als vorbereitenden Naht zu überzeugen. Ich fasse meine Ausführungen in folgenden Sätzen zusammen:

1. Die Klemmnaht, als vorbereitende Naht, kann allenthalben eine ausgedehnte Verwendung finden.

2. Durch die Technik wird die Naht vereinfacht, die Aseptik erhöht, da große Wundflächen rasch abgeschlossen werden können, und außerdem die Arbeit mit den Fingern herabgesetzt wird.

3. Exakte Adaption der Wundränder ist ein ganz besonderer Vorzug, ebenso

4. die Möglichkeit, feinstes Material zu verwenden. 
532 XXI. Schultze, Die Verwend. der Klemmtechnik in der Chirurgie.

\section{Literaturverzeichnis.}

1. Schultze, Die Klemmnaht, eine fremdkörperfreie Naht. Therapeut. Monatshefte 1901.

2. Derselbe, Die Behandlung des statischen Plattfußes mittels des Redressement forcé und der Sehnenplastik. Kongreß für orthopädische Chirurgie 1904.

3. Derselbe, Beitrag zur Magenresektion. Eine Modifikation der Kocherschen Magenresektion. Deutsche Zeitschrift für Chirurgie 1905.

4. Bernhard, Zentralblatt f. Chirurgie 1905. 